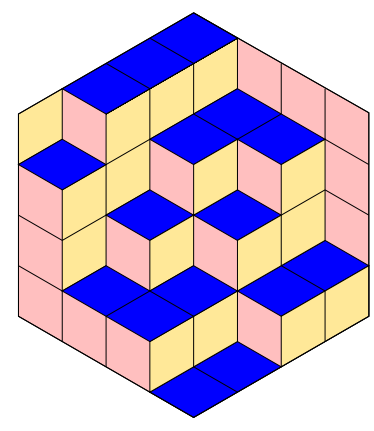

ALGEBRAIC COMBINATORICS

Zachary Hamaker, Brendan Pawlowski \& Bruce E. Sagan

Pattern avoidance and quasisymmetric functions

Volume 3, issue 2 (2020), p. 365-388.

<http://alco.centre-mersenne.org/item/ALCO_2020__3_2_365_0>

(C) The journal and the authors, 2020.

Some rights reserved.

(c) BY This article is licensed under the

Creative Commons ATtribution 4.0 InTERnational License.

http://creativecommons.org/licenses/by/4.0/

Access to articles published by the journal Algebraic Combinatorics on the website http://alco.centre-mersenne.org/ implies agreement with the Terms of Use (http://alco.centre-mersenne.org/legal/).

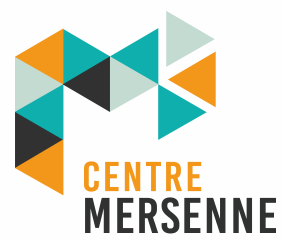

Algebraic Combinatorics is member of the Centre Mersenne for Open Scientific Publishing www.centre-mersenne.org 


\title{
Pattern avoidance and quasisymmetric functions
}

\author{
Zachary Hamaker, Brendan Pawlowski \& Bruce E. Sagan
}

\begin{abstract}
Given a set of permutations $\Pi$, let $\mathfrak{S}_{n}(\Pi)$ denote the set of permutations in the symmetric group $\mathfrak{S}_{n}$ that avoid every element of $\Pi$ in the sense of pattern avoidance. Given a subset $S$ of $\{1, \ldots, n-1\}$, let $F_{S}$ be the fundamental quasisymmetric function indexed by $S$. Our object of study is the generating function $Q_{n}(\Pi)=\sum F_{\operatorname{Des} \sigma}$ where the sum is over all $\sigma \in \mathfrak{S}_{n}(\Pi)$ and Des $\sigma$ is the descent set of $\sigma$. We characterize those $\Pi \subseteq \mathfrak{S}_{3}$ such that $Q_{n}(\Pi)$ is symmetric or Schur nonnegative for all $n$. In the process, we show how each of the resulting $\Pi$ can be obtained from a theorem or conjecture involving more general sets of patterns. In particular, we prove results concerning symmetries, shuffles, and Knuth classes, as well as pointing out a relationship with the arc permutations of Elizalde and Roichman. Various conjectures and questions are mentioned throughout.
\end{abstract}

\section{INTRODUCTION}

Let $\mathfrak{S}_{n}$ be the symmetric group of all permutations of $[n]=\{1, \ldots, n\}$. We view the permutations in $\mathfrak{S}_{n}$ as sequences $\pi=\pi_{1} \ldots \pi_{n}$. Given any sequence $\sigma$ of $k$ distinct integers, its standardization is the permutation $\operatorname{std} \sigma \in \mathfrak{S}_{k}$ obtained by replacing the smallest element of $\sigma$ by 1 , the next smallest by 2 , and so forth. We say that permutation $\sigma$ contains permutation $\pi$ as a pattern if there is a subsequence $\sigma^{\prime}$ of (not necessarily consecutive) elements of $\sigma$ such that std $\sigma^{\prime}=\pi$. For example, $\sigma=5132746$ contains $\pi=231$ because of the subsequence $\sigma^{\prime}=574$. Permutation $\sigma$ avoids $\pi$ if it does not contain $\pi$ as a pattern. Given a set of permutations $\Pi$ we let

$$
\mathfrak{S}_{n}(\Pi)=\left\{\sigma \in \mathfrak{S}_{n} \mid \sigma \text { avoids every } \pi \in \Pi\right\} .
$$

For more information about permutation patterns, see the book of Bóna [3]. Our object is to make a connection between the theory of patterns and the theory of quasisymmetric functions. First we will review some material concerning symmetric functions. Details about symmetric functions and related combinatorics such as the Robinson-Schensted map can be found in the texts of Macdonald [10], Sagan [13], or Stanley [16].

Let $\mathbf{x}=\left\{x_{1}, x_{2}, \ldots\right\}$ be a countably infinite set of commuting variables and consider the algebra of formal power series over the rationals $\mathbb{Q} \llbracket \mathbf{x} \rrbracket$. Consider a monomial $m=x_{i_{1}}^{n_{1}} \ldots x_{i_{k}}^{n_{k}}$. The degree of $m$ is $\sum_{i} n_{i}$, and the degree of any $f \in \mathbb{Q} \llbracket \mathbf{x} \rrbracket$ is the maximum degree of a monomial in $f$ if the maximum exists or infinity otherwise. We

Manuscript received 26th October 2018, revised 7th June 2019, accepted 18th September 2019.

KEYwORDS. Knuth class, pattern avoidance, quasisymmetric function, Schur function, shuffle, symmetric function, Young tableau. 
say that $f$ is symmetric if it is of bounded degree and invariant under permutation of variables. For example

$$
f=x_{1}^{2} x_{2}+x_{1} x_{2}^{2}+x_{1}^{2} x_{3}+x_{1} x_{3}^{2}+x_{2}^{2} x_{3}+x_{2} x_{3}^{2}+\ldots
$$

is symmetric. Let $\mathrm{Sym}_{n}$ denote the vector space of symmetric functions homogeneous of degree $n$. Bases for $\operatorname{Sym}_{n}$ are indexed by partitions of $n$, which are weakly decreasing sequences of positive integers $\lambda=\left(\lambda_{1}, \ldots, \lambda_{k}\right)$ with $\sum_{i} \lambda_{i}=n$. If $\lambda$ is a partition of $n$ then we write $\lambda \vdash n$ or $|\lambda|=n$. The $\lambda_{i}$ are called the parts of $\lambda$.

We will be particularly interested in the important Schur basis for $\mathrm{Sym}_{n}$. Recall that a partition $\lambda=\left(\lambda_{1}, \ldots, \lambda_{k}\right)$ has an associated Young diagram consisting of $k$ leftjustified rows of boxes with $\lambda_{i}$ boxes in row $i$. We will write our diagrams in English notation with the first row on top and often make no distinction between a partition and its diagram. Given $\lambda \vdash n$ then a standard Young tableau (SYT), P, of shape $\lambda$ is obtained by filling the boxes bijectively with the elements of $[n]$ so that rows and columns increase. In a semistandard Young tableau (SSYT), T, of shape $\lambda$ the entries are positive integers distributed so that rows weakly increase and columns strictly increase. We write $\operatorname{SYT}(\lambda)$ or $\operatorname{SSYT}(\lambda)$ for the set of standard or semistandard Young tableaux of shape $\lambda$, respectively. We also write $\operatorname{sh} P=\lambda$ or $\operatorname{sh} T=\lambda$ to indicate that $P$ or $T$ have shape $\lambda$. The Schur function corresponding to $\lambda$ is

$$
s_{\lambda}=\sum_{T} \prod_{i \in T} x_{i}
$$

For example, the set $\operatorname{SSYT}(2,1)$ consists of

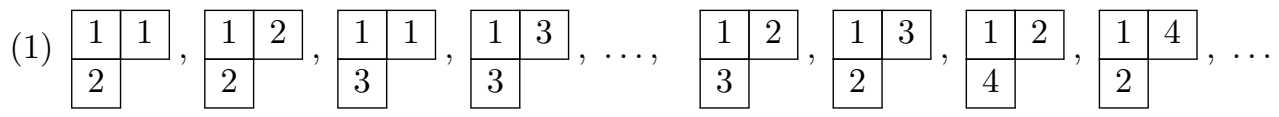

resulting in

$$
s_{(2,1)}=x_{1}^{2} x_{2}+x_{1} x_{2}^{2}+x_{1}^{2} x_{3}+x_{1} x_{3}^{2}+\cdots+2 x_{1} x_{2} x_{3}+2 x_{1} x_{2} x_{4}+\cdots
$$

as the corresponding Schur function. Call a function $f \in \operatorname{Sym}_{n}$ Schur nonnegative if its expansion in the $s_{\lambda}$ basis has nonnegative coefficients.

Quasisymmetric functions refine symmetric functions. Call a formal power series $g$ of bounded degree quasisymmetric if any two monomials $x_{i_{1}}^{n_{1}} \ldots x_{i_{k}}^{n_{k}}$ where $i_{1}<\cdots<i_{k}$ and $x_{j_{1}}^{n_{1}} \ldots x_{j_{k}}^{n_{k}}$ where $j_{1}<\cdots<j_{k}$ have the same coefficient in $g$. By way of illustration,

$$
g=x_{1}^{2} x_{2}+x_{1}^{2} x_{3}+x_{1}^{2} x_{4}+\cdots+x_{2}^{2} x_{3}+x_{2}^{2} x_{4}+\cdots+x_{3}^{2} x_{4}+\ldots
$$

is quasisymmetric, but not symmetric. Clearly every symmetric function is quasisymmetric. Quasisymmetric functions were introduced by Gessel [9] in his work on Stanley's theory of $P$-partitions. They have since found many applications in both enumerative and algebraic combinatorics. We denote by $\mathrm{QSym}_{n}$ the vector space of quasisymmetric functions homogeneous of degree $n$. Bases for this vector space are indexed by compositions of $n$, which are sequences of positive integers $\alpha=\left(\alpha_{1}, \ldots, \alpha_{k}\right)$ summing to $n$. We will use the notations $\alpha \models n$ and $|\alpha|=n$ for compositions of $n$. Greek letters near the beginning of the alphabet will be used for compositions while those near the middle will represent partitions. There is also an important bijection between compositions of $n$ and subsets $S \subseteq[n-1]$ given by

$$
\left(\alpha_{1}, \alpha_{2}, \alpha_{3}, \ldots, \alpha_{k}\right) \mapsto\left\{\alpha_{1}, \alpha_{1}+\alpha_{2}, \alpha_{1}+\alpha_{2}+\alpha_{3}, \ldots, \alpha_{1}+\alpha_{2}+\cdots+\alpha_{k-1}\right\} .
$$

We will sometimes go back and forth between a composition and its associated set without mention. 
The basis we will be using for $\mathrm{QSym}_{n}$ was considered in Gessel's original paper. Given $S \subseteq[n-1]$, the associated fundamental quasisymmetric function is

$$
F_{S}=\sum x_{i_{1}} x_{i_{2}} \ldots x_{i_{n}}
$$

where the sum is over all indices such that $i_{1} \leqslant i_{2} \leqslant \cdots \leqslant i_{n}$ and $i_{s}<i_{s+1}$ if $s \in S$. For example, if $S=\{1\} \subseteq[2]$ then the sum would be over all $x_{i} x_{j} x_{k}$ with $i<j \leqslant k$, which gives

$$
F_{\{1\}}=x_{1} x_{2}^{2}+x_{1} x_{3}^{2}+x_{2} x_{3}^{2}+\cdots+x_{1} x_{2} x_{3}+x_{1} x_{2} x_{4}+x_{1} x_{3} x_{4}+x_{2} x_{3} x_{4}+\cdots
$$

in $\mathrm{QSym}_{3}$. Since $s_{\lambda}$ is symmetric, and hence quasisymmetric, it can be expanded in the $F_{S}$ basis. To do so, we need the descent set of an $S Y T, P$, which is

$$
\text { Des } P=\{i \mid i+1 \text { is in a lower row than } i \text { in } P\} \text {. }
$$

For example, if

$$
P=\begin{array}{|l|l|l|l|}
\hline 1 & 2 & 5 & 9 \\
\hline 3 & 4 & 7 & \multicolumn{1}{|c}{} \\
\cline { 1 - 2 } 6 & 8 & \multicolumn{2}{|c}{} \\
\cline { 1 - 2 } & &
\end{array}
$$

then Des $P=\{2,5,7\}$. Note that if $P$ has shape $\lambda \vdash n$ then Des $P \subseteq[n-1]$.

Theorem 1.1 ([9]). We have

$$
s_{\lambda}=\sum_{P \in \operatorname{SYT}(\lambda)} F_{\operatorname{Des} P} .
$$

Returning to $\lambda=(2,1)$, the two elements of $\operatorname{SYT}(2,1)$ are the fifth and sixth displayed tableaux in (1) with descent sets $\{2\}$ and $\{1\}$ respectively. Therefore $s_{(2.1)}=$ $F_{\{1\}}+F_{\{2\}}$.

To combine permutation patterns and quasisymmetric functions, recall that the descent set of a permutation $\pi=\pi_{1} \ldots \pi_{n}$ is

$$
\text { Des } \pi=\left\{i \mid \pi_{i}>\pi_{i+1}\right\} \subseteq[n-1] .
$$

For example, $\operatorname{Des}(35716824)=\{3,6\}$. Now given a set of patterns $\Pi$ we define the pattern quasisymmetric function

$$
Q_{n}(\Pi)=\sum_{\sigma \in \mathfrak{S}_{n}(\Pi)} F_{\operatorname{Des} \sigma} .
$$

The basic questions we wish to ask about these functions are

(1) When is $Q_{n}(\Pi)$ symmetric for all $n$ ?

(2) In that case, when is $Q_{n}(\Pi)$ Schur nonnegative for all $n$ ?

Note that we are asking about symmetry or Schur nonnegativity not for a single function, but rather for an infinite family of functions.

We start with $\Pi \subseteq \mathfrak{S}_{3}$ and will prove the following theorem for which we will need some preliminaries. If $\{123,321\} \subseteq \Pi$ then, by the Erdös-Szekeres Theorem [8], $\mathfrak{S}_{n}(\Pi)=\varnothing$ for $n \geqslant 5$, which explains the hypothesis on $\Pi$. We use the notation $f^{\lambda}$ for the number of SYT of shape $\lambda$. The transpose of $\lambda$ is the diagram $\lambda^{t}$ obtained by reflecting $\lambda$ in the main diagonal. Then $\lambda_{1}^{t}$ is the number of boxes in the first column of $\lambda$, which is also written as $\ell(\lambda)$ and called the length of $\lambda$. Also, a hook is a partition of the form $\left(a, 1^{b}\right)$ for nonnegative integers $a, b$ where $1^{b}$ denotes the part 1 repeated $b$ times. When using a partition as a subscript we often omit the parentheses. 


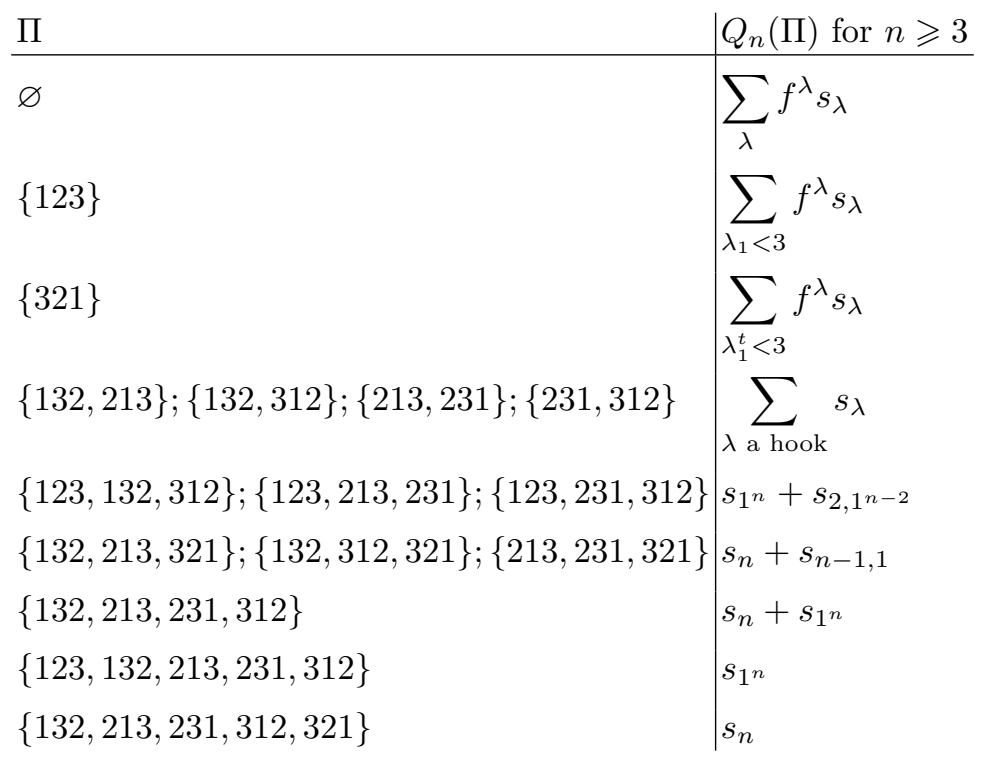

TABLE 1. The $\Pi$ for Theorem 1.2 along with the Schur expansions of $Q_{n}(\Pi)$ where $\lambda \vdash n$

THEOREM 1.2. Suppose $\{123,321\} \nsubseteq \Pi \subseteq \mathfrak{S}_{3}$. The following are equivalent

(1) $Q_{n}(\Pi)$ is symmetric for all $n$.

(2) $Q_{n}(\Pi)$ is Schur nonnegative for all $n$.

(3) $\Pi$ is an entry in Table 1.

We note that it is easy to compute examples to show that for any $\Pi$ not listed in Table 1 we have $Q_{n}(\Pi)$ not being symmetric for some small value of $n$ depending on $\Pi$. Therefore it suffices to show that the $\Pi$ in the table have the claimed Schur expansions. In fact, we will show that these expansions are special cases of more general results or conjectures where $\Pi$ is not restricted to $\mathfrak{S}_{3}$.

The rest of this paper is structured as follows. In the next section we discuss what effect reversal and complementation have on $Q_{n}(\Pi)$ as well as using properties of the Robinson-Schensted correspondence to derive some of the results in Table 1. In Section 3 we show how the quasisymmetric function for a shuffle of two pattern sets can be computed in terms of the ones for each individual component. Shuffles with increasing and decreasing permutations as well as full symmetric groups are used as examples. We define partial shuffles in Section 4 as certain shuffles where the increasing permutation has been removed. We conjecture that in this case $Q_{n}(\Pi)$ has a nice Schur expansion and prove this in a special case. Clearly if $\mathfrak{S}_{n}(\Pi)$ is a union of Knuth classes then $Q_{n}(\Pi)$ is symmetric and Schur nonnegative. In Section 5 we study when this can happen and, in particular, characterize the SYT such that the permutations avoiding its Knuth class have this property. We show in Section 6 that avoiders of the arc permutations of Elizalde and Roichman $[4,5]$ are in bijection with the permutations avoiding a certain set of shuffles. We end with a section of comments and open questions. We should note that several of our results were derived independently by Elizalde and Roichman [7] in the context of products and grid classes. For these, we provide a citation of their work in the corresponding proposition of this article. In this paper, they also find $Q_{n}(\Pi)$ for various sets of patterns not consideered here, for example when $\Pi=\{321,2143,2413\}$. 


\section{Symmetries And the Robinson-Schensted Bijection}

If one views a permutation as a permutation matrix, then the dihedral group $D_{4}$ of the square acts on $\mathfrak{S}_{n}$. We wish to investigate whether this action tells us anything about $Q_{n}(\Pi)$. In particular, if this function is symmetric and one acts on $\Pi$ with a dihedral symmetry then is the new quasisymmetric function symmetric? If so, what is its Schur expansion? As usual, we apply a symmetry to a set by applying it to each member of the set.

First we note that if $Q_{n}(\Pi)$ is a symmetric function then $Q_{n}\left(\Pi^{-1}\right)$ need not be. For example, one can easily check that this is true if $n=3$ and $\Pi=\{132,312\}$. Therefore we can expect the dihedral symmetries preserving symmetry of $Q_{n}$ to form a subgroup of order at most 4 in $D_{4}$. We will now show that such a subgroup exists.

It is easy to describe two of the symmetries in $D_{4}$ directly in terms of the permutations in $\mathfrak{S}_{n}$. The permutation $\pi=\pi_{1} \pi_{2} \ldots \pi_{n}$ has complement $\pi^{c}=n+1-$ $\pi_{1}, n+1-\pi_{2}, \ldots, n+1-\pi_{n}$ where we have inserted commas between the elements for readability. Its reversal is $\pi^{r}=\pi_{n} \pi_{n-1} \ldots \pi_{1}$. For example, if $\pi=35716824$ then $\pi^{c}=64283175$ and $\pi^{r}=42861753$. Complementation and reversal are two of the reflections in $D_{4}$ and so $\pi^{r c}=\pi^{c r}$ is the permutation whose matrix is obtained by rotating the matrix of $\pi$ by $180^{\circ}$. To state our first dihedral symmetry result, recall that the transpose $\lambda^{t}$ of a Young diagram $\lambda$ is gotten by reflecting $\lambda$ in its main diagonal. We use the same notation for tableaux.

Proposition 2.1. If $Q_{n}(\Pi)$ is symmetric then so is $Q_{n}\left(\Pi^{c}\right)$. In particular, if $Q_{n}(\Pi)=$ $\sum_{\lambda} c_{\lambda} s_{\lambda}$ for certain coefficients $c_{\lambda}$ then

$$
Q_{n}\left(\Pi^{c}\right)=\sum_{\lambda} c_{\lambda} s_{\lambda^{t}}
$$

Proof. Using Theorem 1.1 we can write

$$
\sum_{\sigma \in \mathfrak{S}_{n}(\Pi)} F_{\operatorname{Des} \sigma}=Q_{n}(\Pi)=\sum_{\lambda} c_{\lambda} s_{\lambda}=\sum_{\lambda} c_{\lambda} \sum_{P \in \operatorname{SYT}(\lambda)} F_{\operatorname{Des} P} .
$$

Note that for any permutation Des $\pi^{c}=[n-1]-\operatorname{Des} \pi$ and $\mathfrak{S}_{n}\left(\Pi^{c}\right)=\left(\mathfrak{S}_{n}(\Pi)\right)^{c}$. Also, for any standard Young tableau Des $P^{t}=[n-1]-$ Des $P$. Using these facts and the previous displayed equation gives

$$
Q_{n}\left(\Pi^{c}\right)=\sum_{\sigma \in \mathfrak{S}_{n}(\Pi)} F_{[n-1]-\operatorname{Des} \sigma}=\sum_{\lambda} c_{\lambda} \sum_{P \in \operatorname{SYT}(\lambda)} F_{[n-1]-\operatorname{Des} P}=\sum_{\lambda} c_{\lambda} s_{\lambda^{t}}
$$

as desired.

In order to deal with reversals, we will need some background about the RobinsonSchensted map $[12,14]$. This is a bijection

$$
\mathrm{RS}: \mathfrak{S}_{n} \rightarrow \bigcup_{\lambda \vdash n} \operatorname{SYT}(\lambda) \times \operatorname{SYT}(\lambda) .
$$

If $\operatorname{RS}(\pi)=(P, Q)$ then we will write $P=P(\pi)$ and $Q=Q(\pi)$ and call $P$ and $Q$ the $P$-tableau and $Q$-tableau of $\pi$, respectively. If $\pi=\pi_{1} \ldots \pi_{n}$ then $P$ is constructed using an operation called insertion that sequentially inserts $\pi_{1}, \ldots, \pi_{n}$ to form $P$. After the $k$ th insertion, a $k$ is placed in $Q$ so that one maintains $\operatorname{sh} P=\operatorname{sh} Q$ at all times. Although we are using $Q$ in both the notation $Q(\pi)$ and $Q_{n}(\Pi)$ the two different concepts should be distinguishable by context and the fact that the latter has a subscript while the former does not.

We put an equivalence relation on $\mathfrak{S}_{n}$ by declaring that $\pi$ and $\sigma$ are Knuth equivalent, written $\pi \sim \sigma$, if $P(\pi)=P(\sigma)$. Given an SYT $P$, the corresponding Knuth class is

$$
K(P)=\{\pi \mid P(\pi)=P\} .
$$


Given a partition $\lambda$ it will also be convenient to define an associated Knuth aggregate by

$$
K(\lambda)=\{\pi \mid \operatorname{sh} P(\pi)=\lambda\}=\bigcup_{\operatorname{sh}(P)=\lambda} K(P) .
$$

We will need the following properties of the map RS. For proofs of these results, see [16].

Theorem 2.2. Suppose $\operatorname{RS}(\pi)=(P, Q)$.

(1) $\operatorname{Des} \pi=\operatorname{Des} Q$.

(2) If $\operatorname{sh} P=\lambda$ then $\lambda_{1}$ is the length of a longest increasing subsequence of $\pi$.

(3) $P\left(\pi^{r}\right)=(P(\pi))^{t}$.

(4) $\operatorname{RS}\left(\pi^{-1}\right)=(Q, P)$.

These results have interesting implications for our quasisymmetric functions. To state them conveniently we will use the notation $F_{\pi}=F_{\text {Des } \pi}$ for a permutation $\pi$. And for a set $\Pi$ of permutations we define $F_{\Pi}=\sum_{\pi \in \Pi} F_{\pi}$.

Lemma 2.3. Suppose $P \in \operatorname{SYT}(\lambda)$.

(1) $F_{K(P)}=s_{\lambda}$.

(2) $F_{K(\lambda)}=f^{\lambda} s_{\lambda}$.

Proof. Using the fact that RS is a bijection, Theorem 1.1, and Theorem 2.2 (1) we have

$$
F_{K(P)}=\sum_{\pi \in K(P)} F_{\operatorname{Des} \pi}=\sum_{Q \in \operatorname{SYT}(\lambda)} F_{\operatorname{Des} Q}=s_{\lambda}
$$

which is (1). For (2), we use (1) to write

$$
F_{K(\lambda)}=\sum_{\operatorname{sh}(P)=\lambda} F_{K(P)}=\sum_{\operatorname{sh}(P)=\lambda} s_{\lambda}=f^{\lambda} s_{\lambda}
$$

as desired.

We will now prove that Proposition 2.1 continues to hold if complement is replaced by reversal.

Proposition 2.4. If $Q_{n}(\Pi)$ is symmetric then so is $Q_{n}\left(\Pi^{r}\right)$. In particular, if $Q_{n}(\Pi)=$ $\sum_{\lambda} c_{\lambda} s_{\lambda}$ for certain coefficients $c_{\lambda}$ then

$$
Q_{n}\left(\Pi^{r}\right)=\sum_{\lambda} c_{\lambda} s_{\lambda^{t}}
$$

Proof. For each partition $\lambda$ pick a tableau $P_{\lambda} \in \operatorname{SYT}(\lambda)$. Then using Lemma 2.3 (1) we have

$$
\sum_{\sigma \in \mathfrak{S}_{n}(\Pi)} F_{\operatorname{Des} \sigma}=Q_{n}(\Pi)=\sum_{\lambda} c_{\lambda} s_{\lambda}=\sum_{\lambda} c_{\lambda} F_{K\left(P_{\lambda}\right)} .
$$

Combining this with Theorem 2.2 (3) yields

$$
Q_{n}\left(\Pi^{r}\right)=\sum_{\sigma \in \mathfrak{S}_{n}(\Pi)} F_{\operatorname{Des} \sigma^{r}}=\sum_{\lambda} c_{\lambda} F_{K\left(\left(P_{\lambda}\right)^{t}\right)}=\sum_{\lambda} c_{\lambda} s_{\lambda^{t}}
$$

which is what we wished to prove.

As an immediate corollary of Propositions 2.1 and 2.4 we get the following.

Proposition 2.5 (cf. [7, Lemma 8.1]). If $Q_{n}(\Pi)$ is symmetric then so is $Q_{n}\left(\Pi^{c r}\right)$. In particular, $Q_{n}\left(\Pi^{c r}\right)=Q_{n}(\Pi)$. 
As an application of these ideas, we will now verify the expansions in the first three and last three rows of Table 1. For the first row, we use Lemma 2.3 (2) and the fact that $\mathrm{RS}$ is a bijection to write

$$
Q_{n}(\varnothing)=\sum_{\sigma \in \mathfrak{S}_{n}} F_{\sigma}=\sum_{\lambda \vdash n} F_{K(\lambda)}=\sum_{\lambda \vdash n} f^{\lambda} s_{\lambda} .
$$

The next two rows are special cases of the following result. In it and the sequel we eliminate the braces when writing out $Q_{n}(\Pi)$ for a specific $\Pi$. We will also use the notation

$$
\iota_{k}=12 \ldots k
$$

and

$$
\delta_{k}=k \ldots 21
$$

for the increasing and decreasing permutations of length $k$.

Proposition 2.6. For any $k \geqslant 1$ we have

$$
Q_{n}\left(\iota_{k}\right)=\sum_{\lambda_{1}<k} f^{\lambda} s_{\lambda}
$$

and

$$
Q_{n}\left(\delta_{k}\right)=\sum_{\lambda_{1}^{t}<k} f^{\lambda} s_{\lambda}
$$

Proof. By either Proposition 2.1 or Proposition 2.4, it suffices to prove the first statement. Note that $\sigma \in \mathfrak{S}_{n}\left(\iota_{k}\right)$ if and only if the longest increasing subsequence of $\sigma$ has length less than $k$. Now using Theorem 2.2 (2), Lemma 2.3 (2) and the bijectivity of RS we obtain

$$
Q_{n}\left(\iota_{k}\right)=\sum_{\sigma \in \mathfrak{S}_{n}\left(\iota_{k}\right)} F_{\sigma}=\sum_{\lambda_{1}<k} F_{K(\lambda)}=\sum_{\lambda_{1}<k} f^{\lambda} s_{\lambda}
$$

which is the desired result.

As far as the last three rows of Table 1, the reader will find it easy to prove the following result so the proof is omitted.

Proposition 2.7. For $\Pi=\mathfrak{S}_{k}-\left\{\iota_{k}, \delta_{k}\right\}$ we have

$$
Q_{n}(\Pi)= \begin{cases}\sum_{\lambda \vdash n} f^{\lambda} s_{\lambda} & \text { for } n<k, \\ s_{n}+s_{1^{n}} & \text { for } n \geqslant k .\end{cases}
$$

For $\Pi=\mathfrak{S}_{k}-\left\{\delta_{k}\right\}$ we have

$$
Q_{n}(\Pi)= \begin{cases}\sum_{\lambda \vdash n} f^{\lambda} s_{\lambda} & \text { for } n<k, \\ s_{1^{n}} & \text { for } n \geqslant k .\end{cases}
$$

For $\Pi=\mathfrak{S}_{k}-\left\{\iota_{k}\right\}$ we have

$$
Q_{n}(\Pi)= \begin{cases}\sum_{\lambda \vdash n} f^{\lambda} s_{\lambda} & \text { for } n<k, \\ s_{n} & \text { for } n \geqslant k .\end{cases}
$$




\section{Shuffles}

In this section we will show that several of the entries in Table 1 can be explained using shuffles of permutations. It turns out that under very general conditions, shuffling preserves $Q_{n}$ being symmetric, and perhaps Schur nonnegative as well.

If $\pi=\pi_{1} \ldots \pi_{m}$ and $\sigma=\sigma_{1} \ldots \sigma_{n}$ are sequences on distinct elements on disjoint alphabets then their shuffle set is

$$
\pi \varpi \sigma=\left\{\tau=\tau_{1} \ldots \tau_{m+n} \mid \pi \text { and } \sigma \text { are subsequences of } \tau\right\} .
$$

For example

$$
13 \amalg 42=\{1342,1432,1423,4132,4123,4213\} .
$$

If $\pi \in \mathfrak{S}_{m}$ and $\sigma \in \mathfrak{S}_{n}$ then we define $\pi ш \sigma=\pi \uplus(\sigma+m)$ where $\sigma+m$ is the sequence obtained by adding $m$ to each element of $\sigma$. To illustrate

$$
12 \text { ш } 21=12 \amalg 43=\{1243,1423,1432,4123,4132,4312\} .
$$

We shuffle sets of permutations as expected, namely

$$
\Pi ш \Pi^{\prime}=\bigcup_{\pi \in \Pi, \pi^{\prime} \in \Pi^{\prime}} \pi ш \pi^{\prime} .
$$

To prove the next result, it will be useful to have a notation for the permutations in $\mathfrak{S}_{n}$ that contain a pattern in $\Pi$, which will be

$$
\overline{\mathfrak{S}}_{n}(\Pi)=\mathfrak{S}_{n}-\mathfrak{S}_{n}(\Pi) \text {. }
$$

We will also use $s_{1}$ as shorthand for the Schur function $s_{(1)}$.

TheOREM 3.1. For any sets of nonempty permutations $\Pi$ and $\Pi^{\prime}$ and any $n \geqslant 0$,

$$
Q_{n}\left(\Pi \amalg \Pi^{\prime}\right)=Q_{n}\left(\Pi^{\prime}\right)+\sum_{k=0}^{n-1} Q_{k}(\Pi)\left[s_{1} Q_{n-k-1}\left(\Pi^{\prime}\right)-Q_{n-k}\left(\Pi^{\prime}\right)\right] .
$$

Proof. We first show that

$$
\overline{\mathfrak{S}}_{n}\left(\Pi ш \Pi^{\prime}\right)=\bigcup_{k=1}^{n-1} \overline{\mathfrak{S}}_{k}(\Pi) ш \overline{\mathfrak{S}}_{n-k}\left(\Pi^{\prime}\right) .
$$

If $\tau \in \overline{\mathfrak{S}}_{n}\left(\Pi ш \Pi^{\prime}\right)$ then $\tau$ contains $\pi ш \pi^{\prime}$ for some $\pi \in \Pi$ and $\pi^{\prime} \in \Pi^{\prime}$. Let $\tau^{a}$ and $\tau^{b}$ be the copies of $\pi$ and $\pi^{\prime}$, respectively, in $\tau$. This implies $\max \tau^{a}<\min \tau^{b}$. Then the restriction of $\tau$ to the elements of $[k]$ where $k=\max \tau^{a}$ is an element of $\overline{\mathfrak{S}}_{k}(\Pi)$, similarly restricting to $[n]-[k]$ gives an element of $\overline{\mathfrak{S}}_{n-k}\left(\Pi^{\prime}\right)+k$. Thus $\tau \in \overline{\mathfrak{S}}_{k}(\Pi) \amalg \overline{\mathfrak{S}}_{n-k}\left(\Pi^{\prime}\right)$. The reverse inclusion is proven similarly.

The Malvenuto-Reutenauer algebra [11], MR, is the set of formal $\mathbb{Q}$-linear combinations of permutations with product given by shuffle. The map $\Phi:$ MR $\rightarrow$ QSym given by $\pi \mapsto F_{\pi}$ is a homomorphism. Define $A_{k}=\overline{\mathfrak{S}}_{k}(\Pi) ш \overline{\mathfrak{S}}_{n-k}\left(\Pi^{\prime}\right)$. Applying $\Phi$ to both sides of the summation identity implied by (2) and then using the Principle of Inclusion-Exclusion gives

$$
\Phi\left(\overline{\mathfrak{S}}_{n}\left(\Pi \varpi \Pi^{\prime}\right)\right)=\sum_{p=1}^{n-1}(-1)^{p-1} \sum_{1 \leqslant k_{1}<\cdots<k_{p} \leqslant n-1} \Phi\left(A_{k_{1}} \cap \cdots \cap A_{k_{p}}\right) .
$$

A proof similar to the one for (2) shows that if $k<\ell$ then

$$
A_{k} \cap A_{\ell}=\overline{\mathfrak{S}}_{k}(\Pi) ш \mathfrak{S}_{\ell-k} ш \overline{\mathfrak{S}}_{n-\ell}\left(\Pi^{\prime}\right) \text {. }
$$

Indeed, if $\sigma \in A_{k} \cap A_{\ell}$ then, since $\sigma \in A_{k}$, the smallest $k$ elements of $\sigma$ contain a copy of $\pi \in \Pi$. Since $\ell>k$ it is automatic that the smallest $\ell$ elements of $\sigma$ contain the same copy of $\pi$. Similarly we have that the last $n-\ell$ elements of $\sigma$ must contain a copy of some element of $\Pi^{\prime}$. And it is easy to check that the other elements can be 
arranged arbitrarily so that $\sigma \in \overline{\mathfrak{S}}_{k}(\Pi) ш \mathfrak{S}_{\ell-k} ш \overline{\mathfrak{S}}_{n-\ell}\left(\Pi^{\prime}\right)$. The reader should now be able to fill in the details of the reverse containment.

From (4) it follows that if $k_{1}<\cdots<k_{p}$, then $A_{k_{1}} \cap \cdots \cap A_{k_{p}}=A_{k_{1}} \cap A_{k_{p}}$. Applying this observation to (3) and then using the fact that $\sum_{k}(-1)^{k}\left(\begin{array}{l}n \\ k\end{array}\right)=\delta_{n, 0}$ gives

$$
\begin{aligned}
\Phi\left(\widetilde{\mathfrak{S}}_{n}\left(\Pi \varpi \Pi^{\prime}\right)\right) & =\sum_{k=1}^{n-1} \Phi\left(A_{k}\right)+\sum_{1 \leqslant k<\ell \leqslant n-1} \Phi\left(A_{k} \cap A_{\ell}\right) \sum_{p=2}^{n-1}(-1)^{p-1}\left(\begin{array}{c}
\ell-k-1 \\
p-2
\end{array}\right) \\
& =\sum_{k=1}^{n-1} \Phi\left(A_{k}\right)-\sum_{k=1}^{n-2} \Phi\left(A_{k} \cap A_{k+1}\right) .
\end{aligned}
$$

Now using the definition of $A_{k}$, equation (4), and the fact that $\Phi$ is a homomorphism yields

$$
\begin{aligned}
\Phi\left(\overline{\mathfrak{S}}_{n}\left(\Pi \varpi \Pi^{\prime}\right)\right) & =\sum_{k=1}^{n-1} \Phi\left(\overline{\mathfrak{S}}_{k}(\Pi) ш \overline{\mathfrak{S}}_{n-k}\left(\Pi^{\prime}\right)\right)-\sum_{k=1}^{n-2} \Phi\left(\overline{\mathfrak{S}}_{k}(\Pi) ш \mathfrak{S}_{1} ш \overline{\mathfrak{S}}_{n-k-1}\left(\Pi^{\prime}\right)\right) \\
& =\sum_{k=1}^{n-1} \Phi\left(\overline{\mathfrak{S}}_{k}(\Pi)\right) \Phi\left(\overline{\mathfrak{S}}_{n-k}\left(\Pi^{\prime}\right)\right)-\sum_{k=1}^{n-2} \Phi\left(\overline{\mathfrak{S}}_{k}(\Pi)\right) s_{1} \Phi\left(\overline{\mathfrak{S}}_{n-k-1}\left(\Pi^{\prime}\right)\right) .
\end{aligned}
$$

Write $\Phi\left(\overline{\mathfrak{S}}_{n}(\Pi)\right)=\Phi\left(\mathfrak{S}_{n}\right)-Q_{n}(\Pi)=s_{1}^{n}-Q_{n}(\Pi)$ and expand the binomials. All terms will cancel except the products involving at most one factor of $s_{1}$ from the first sum and $Q_{k}(\Pi) s_{1} Q_{n-k-1}\left(\Pi^{\prime}\right)$ from the second. Rearranging terms gives the formula in the theorem once one takes account of the fact that the initial $Q_{n}\left(\Pi^{\prime}\right)$ summand cancels into the $k=0$ term of the sum.

We note that this theorem takes a nice form when expressed in terms of generating functions. In particular, if one lets

$$
Q(\Pi)=\sum_{n=0}^{\infty} Q_{n}(\Pi) t^{n}
$$

then the previous result becomes the following.

COROllary 3.2. For any sets of permutations $\Pi$ and $\Pi^{\prime}$,

$$
Q\left(\Pi ш \Pi^{\prime}\right)=Q(\Pi)+Q\left(\Pi^{\prime}\right)+\left(t s_{1}-1\right) Q(\Pi) Q\left(\Pi^{\prime}\right) .
$$

We also have the following immediate corollary of Theorem 3.1

COROLlary 3.3. For any sets of nonempty permutations $\Pi$ and $\Pi^{\prime}$, if $Q_{n}(\Pi)$ and $Q_{n}\left(\Pi^{\prime}\right)$ are symmetric for all $n$ then then same is true of $Q_{n}\left(\Pi \sqcup \Pi^{\prime}\right)$.

Unfortunately, the theorem does not show that Schur nonnegativity is preserved. However computer evidence supports this conjecture.

CONJECTURE 3.4. For any sets of nonempty permutations $\Pi$ and $\Pi^{\prime}$, if $Q_{n}(\Pi)$ and $Q_{n}\left(\Pi^{\prime}\right)$ are Schur nonnegative for all $n$ then then same is true of $Q_{n}\left(\Pi \sqcup \Pi^{\prime}\right)$.

Although we can not do so in general, we can still derive Schur nonnegativity under certain circumstances.

Lemma 3.5. Suppose that, for all $n \geqslant 0$

$$
G_{n}=\sum_{\lambda \vdash n} c_{\lambda} s_{\lambda}
$$


for certain constants $c_{\lambda}$. Define, for all $n \geqslant 1$, a symmetric function $G_{n}^{\prime}$ and constants $d_{\lambda}$ by

(1) We have

$$
G_{n}^{\prime}=s_{1} G_{n-1}-G_{n}=\sum_{\lambda \vdash n} d_{\lambda} s_{\lambda}
$$

$$
d_{\lambda}=\left(\sum_{\lambda^{-}} c_{\lambda^{-}}\right)-c_{\lambda}
$$

where $\lambda^{-}$ranges over all diagrams obtained by removing a box from the diagram of $\lambda$

(2) If $c_{\lambda} \leqslant \sum_{\lambda^{-}} c_{\lambda^{-}}$for all $\lambda \vdash n$ then $G_{n}^{\prime}$ is Schur-nonnegative.

Proof. Clearly (2) follows from (1). And (1) itself follows easily from the Pieri rule for multiplying Schur functions.

As an application, we will consider shuffles with $\iota_{k}, \delta_{k}$, and $\mathfrak{S}_{k}$.

COROLlaRY 3.6. If $Q_{n}(\Pi)$ is Schur-nonnegative for all $n \geqslant 0$ then so are the following: $Q_{n}\left(\Pi \amalg \iota_{k}\right), Q_{n}\left(\Pi \varpi \delta_{k}\right)$, and $Q_{n}\left(\Pi \amalg \mathfrak{S}_{k}\right)$.

Proof. We will only prove first statement as the others are similar. Recall that the Littlewood-Richardson rule expresses the product of two Schur functions as a nonnegative linear combination of Schur functions. Then in view of Theorem 3.1, it suffices to show that condition (2) of Lemma 3.5 is satisfied where $G_{n}=Q_{n}\left(\iota_{k}\right)$. By Proposition 2.6, if $\lambda_{1} \geqslant k$ then $c_{\lambda}=0$ and so the inequality is immediate. On the other hand, if $\lambda_{1}<k$ then the same is true of all $\lambda^{-}$. In this case

$$
c_{\lambda}=f^{\lambda}=\sum_{\lambda^{-}} f^{\lambda^{-}}=\sum_{\lambda^{-}} c_{\lambda^{-}}
$$

and we are done.

Now we can verify another four entries in Table 1.

ThEOREM 3.7. For $n \geqslant 2$ we have

$$
Q_{n}(123,132,312)=Q_{n}(123,213,231)=s_{1^{n}}+s_{2,1^{n-2}}
$$

and

$$
Q_{n}(132,312,321)=Q_{n}(213,231,321)=s_{n}+s_{n-1,1} .
$$

Proof. By Propositions 2.1 and 2.4, we only need to prove the statement for one of the four sets of permutations. Consider $\{123,132,312\}=\{12\} \amalg\{1\}$ and note that $Q_{k}(1)=\delta_{k, 0}$ where we are using the Kronecker delta. Then for any $\Pi$, Theorem 3.1 gives $Q_{n}(\Pi \varpi 1)=s_{1} Q_{n-1}(\Pi)$. In particular, using Proposition 2.6 and the Pieri rule

$$
Q_{n}(12 ш 1)=s_{1} Q_{n-1}(12)=s_{1} s_{1^{n-1}}=s_{1^{n}}+s_{2,1^{n-2}}
$$

as desired.

\section{Partial shuffles}

We now wish to study and generalize $Q_{n}(132,213)$ (and, by symmetry, $Q_{n}(231,312)$ ). We will do this using a new concept that we call a partial shuffle. First, however, we will derive the quasisymmetric function for $\{132,213\}$ itself.

There is a well-known characterization of the permutations in $\mathfrak{S}_{n}(132,213)$ as those which are reverse layered; see, for example, the paper of Simion and Schmidt [15]. A permutation in $\mathfrak{S}_{n}$ is reverse layered if it is of the form

$$
\pi=a, a+1, \ldots, n, b, b+1, \ldots, a-1, c, c+1, \ldots, b-1, \ldots
$$


For certain $a>b>c>\cdots>0$. We are using the term "reverse" because in this case $\pi^{r}$ is what is usually called a layered permutation. Also, let

$$
\mathcal{H}_{n}=\{\lambda \vdash n \mid \lambda \text { is a hook }\}
$$

and $\operatorname{SYT}\left(\mathcal{H}_{n}\right)$ be the set of tableaux $P$ so that $\operatorname{sh}(P) \in \mathcal{H}_{n}$.

Proposition 4.1 ([7, Corollary 4.11]). We have

$$
Q_{n}(132,213)=Q_{n}(231,312)=\sum_{\lambda \in \mathcal{H}_{n}} s_{\lambda}
$$

Proof. By symmetry, it suffices to prove that $Q_{n}(132,213)$ is equal to the sum. From the description (5) it is clear that the map $\pi \mapsto$ Des $\pi$ gives a bijection $\mathfrak{S}_{n}(132,213) \rightarrow$ $2^{[n-1]}$. Thus

$$
Q_{n}(132,213)=\sum_{S \subseteq[n-1]} F_{S} .
$$

We will be done by Lemma 2.3 (1) if we can show that the map $P \mapsto \operatorname{Des} P$ is a bijection $\operatorname{SYT}\left(\mathcal{H}_{n}\right) \rightarrow 2^{[n-1]}$. But is is easy to see that this map has an inverse. In particular, given $S \subseteq[n-1]$ we construct the hook tableau whose first column has elements $\{1\} \cup(S+1)$ where $S+1 \subset[n]$ is obtained by adding one to each element of $S$.

To try and generalize the previous result, note that $\{132,213\}=(13 \amalg 2)-\{123\}$. We will put a hat on an element of a permutation to indicate the sequence obtained by removing that element. For example, $3 \hat{2} 41=341$. Now define the partial shuffle

$$
(12 \ldots \widehat{n-1} n) \varpi(n-1)=[(12 \ldots \widehat{n-1} n) \amalg(n-1)]-\left\{\iota_{n}\right\} .
$$

To illustrate $13 \varpi 2=\{132,213\}$ and $124 \square 3=\{1243,1324,3124\}$.

For the generalization of hook diagrams, let $(i, j)$ denote the box of a Young diagram in row $i$ and column $j$. And if $P$ is a Young tableau then $P_{i, j}$ will be the entry of $P$ in box $(i, j)$. We will consider the set of onlarged hooks

$$
\mathcal{H}_{n, j}=\{\lambda \vdash n \mid(2, j) \notin \lambda\} .
$$

Note $\mathcal{H}_{n, 2}=\mathcal{H}_{n}$. The following conjecture has Proposition 4.1 as the special case $j=3$.

Conjecture 4.2. For $j \geqslant 3$ we have

$$
Q_{n}((12 \ldots \widehat{j-1} j) \varpi(j-1))=\sum_{\lambda \in \mathcal{H}_{n, j-1}} f^{\bar{\lambda}} s_{\lambda}
$$

where $\bar{\lambda}$ is $\lambda$ with $\lambda_{1}$ replaced by $\min \left\{\lambda_{1}, j-2\right\}$.

This conjecture has recently been proved by Bloom and Sagan [2]. Here we content ourselves with making some progress on the case $j=4$. It follows from Theorem $2.2(2)$ and (3) that if $\pi$ avoids $\delta_{m}$ then $P(\pi)$ has less than $m$ rows. In general it is not true that if $Q_{n}(\Pi)=\sum_{\lambda} c_{\lambda} s_{\lambda}$ then we have $Q_{n}\left(\Pi \cup\left\{\delta_{m}\right\}\right)$ is the same sum restricted to $\lambda$ with $\ell(\lambda)<m$. As an example from Theorem 1.2, $Q_{n}(231,312)$ is Schur non-negative but $Q_{n}(231,312,321)$ is not even symmetric for all $n$. However, this property seems to be enjoyed in the context of partial shuffles.

Conjecture 4.3. For $j \geqslant 3$ let $\Pi=(12 \ldots \widehat{j-1} j) \overline{(j-1)}$. Now for $m \geqslant 2$ we have

$$
Q_{n}\left(\Pi \cup\left\{\delta_{m}\right\}\right)=\sum_{\lambda} f^{\bar{\lambda}} s_{\lambda}
$$

where the sum is over all $\lambda \in \mathcal{H}_{n, j-1}$ such that $\ell(\lambda)<m$, and $\bar{\lambda}$ is $\lambda$ with $\lambda_{1}$ replaced by $\min \left\{\lambda_{1}, j-2\right\}$. 
Note that Conjecture 4.3 implies Conjecture 4.2 by letting $m \rightarrow \infty$. We can give a proof of the case $j=3$ of Conjecture 4.3. In fact, a slight modification of the proof of Proposition 4.1 will work since the bijections used there can be restricted to layered permutations with fewer than $m$ layers and hook tableaux with fewer than $m$ rows, both mapping onto the subsets of $[n-1]$ with fewer than $m$ elements. This proves the following.

Proposition 4.4 ([7, Corollary 4.11]). For $m \geqslant 2$ we have

$$
Q_{n}\left(132,213, \delta_{m}\right)=\sum_{\lambda} s_{\lambda}
$$

where the sum is over all $\lambda \in \mathcal{H}_{n}$ such that $\ell(\lambda)<m$. In particular

$$
Q_{n}(132,213,321)=s_{n}+s_{n-1,1}
$$

and by symmetry

$$
Q_{n}(123,231,312)=s_{1^{n}}+s_{2,1^{n-2}} .
$$

We will now prove Conjecture 4.3 in the special case $j=m=4$, that is, we will consider

$$
\Pi=\{1243,1324,3124,4321\} .
$$

Given $\sigma \in \mathfrak{S}_{n}$ we will write its descent set in increasing order Des $\sigma=\left\{d_{1}<d_{2}<\right.$ $\left.\cdots<d_{\ell}\right\}$. We also let $d_{0}=0$ and $d_{\ell+1}=n$ when convenient. Let $\alpha(\sigma)$ denote the corresponding descent composition so that $\alpha_{i}=d_{i}-d_{i-1}$ for $1 \leqslant i \leqslant \ell+1$. The increasing run of $\sigma$ corresponding to $\alpha_{i}$ is the factor of $\sigma$ consisting of the elements with indices between $d_{i-1}+1$ and $d_{i}$ inclusive. For example, if $\sigma=561342$ then $\operatorname{Des} \sigma=\{2,5\}, \alpha(\sigma)=(2,3,1)$ and the increasing runs of $\sigma$ are 56, 134, and 2. We will need the following description of the increasing runs of a $\sigma$ avoiding 1243 and 3124 . It is analagous to the reverse layered description of the permutations $\sigma$ which avoid $\{132,213\}$, which is equivalent to the increasing runs of $\sigma$ being consecutive integers.

LEMMA 4.5. If $\sigma$ avoids 1243 and 3124 then every increasing run of $\sigma$ of length at least two has the form

$$
a, b, b+1, b+2, \ldots, b+k
$$

for some $a<b$ and some $k \geqslant 0$.

Proof. The result is trivial if the the length of the run is two, so assume it has length at least three. Let $d$ be the maximum (and hence, last) element of the run. Suppose, towards a contradiction, that not every integer in the interval $[b, d]$ appears in this run. In particular, suppose that there is $c$ with $b<c<d$ that is not in the run. Now either $c$ comes before the run so that $\sigma$ contains the subsequence $c a b d$, or after the run so that $\sigma$ contains the subsequence $a b d c$. This gives a contradiction in that $\sigma$ contains either a copy of 3124 or 1243 .

We now define two maps that in certain cases, because of the lemma, will be inverses. The contraction at index $j$ of $\sigma \in \mathfrak{S}_{n}$ is the permutation $c_{j}(\sigma) \in \mathfrak{S}_{n-1}$ obtained by removing $\sigma_{j}$ and then standardizing what remains. The expansion at index $j$ is the permutation $e_{j}(\sigma) \in \mathfrak{S}_{n+1}$ obtained by increasing all the elements of $\sigma$ greater than $\sigma_{j}$ by one and then inserting $\sigma_{j}+1$ directly after $\sigma_{j}$ in the result. Continuing our example above with $\sigma=561342$, we have $c_{5}(561342)=45132$ and also $e_{4}(45132)=561342=\sigma$. To state the next result it will be convenient to have the notation.

$$
A(\alpha, n)=\left\{\sigma \in \mathfrak{S}_{n}(1243,1324,3124,4321) \mid \alpha(\sigma)=\alpha\right\} .
$$


Proposition 4.6. Let $\alpha \models n$ have a part $\alpha_{i} \geqslant 3$ and let $j=\alpha_{1}+\alpha_{2}+\cdots+\alpha_{i}$. Also let $\alpha^{\prime}=n-1$ be the composition obtained from $\alpha$ by replacing $\alpha_{i}$ by $\alpha_{i}-1$. Then contraction and expansion restrict to maps $c_{j}: A(\alpha, n) \rightarrow A\left(\alpha^{\prime}, n-1\right)$ and $e_{j-1}: A\left(\alpha^{\prime}, n-1\right) \rightarrow A(\alpha, n)$ that are inverses of each other.

Proof. We first show that $c_{j}$ is well defined. Suppose $\sigma \in A(\alpha, n)$. Since $\alpha_{i} \geqslant 3$ we have, by Lemma 4.5 , that $\sigma_{j}=\sigma_{j-1}+1$. It follows that removing $\sigma_{j}$ and standardizing will produce a descent at position $j-1$. And since $c_{j}(\sigma)$ comes from standardizing a subpermutation of $\sigma$, it must still avoid the permutations in question. Therefore $c_{j}(\sigma) \in A\left(\alpha^{\prime}, n-1\right)$ as desired.

The fact that $e_{j-1}(\tau)=\sigma$ has the correct descent composition is similar to what was done for $c_{j}(\sigma)$. To show that $\sigma$ still avoids the four patterns, assume to the contrary that it contains a copy $\kappa$ of one of them. Then $\kappa$ must contain both $\sigma_{j-1}$ and $\sigma_{j}=\sigma_{j-1}+1$ because, if not, then $\kappa$ is also in $\tau$ (possibly obtained by replacing $\sigma_{j}$ by $\left.\tau_{j-1}\right)$. Since $\sigma_{j}=\sigma_{j-1}+1$ these two elements of $\kappa$ must correspond to consecutive, increasing elements in the pattern, so the only option is that they become the one and two in either 1243 or 3124 . But $\alpha_{i} \geqslant 3$ so that $\sigma_{j-1}$ is not the first element of its run. Then replacing $\sigma_{j-1} \sigma_{j}$ by $\tau_{j-2} \tau_{j-1}$ gives a copy of the forbidden pattern in $\tau$, a contradiction.

We now show that the two maps are inverses. For any permutation $\tau$ we have $c_{j} e_{j-1}(\tau)=\tau$. On the other hand, if $\sigma \in A(\alpha, n)$ then, again using the fact that $\sigma_{j}=\sigma_{j-1}+1$, we must have $e_{j-1} c_{j}(\sigma)=\sigma$ because the element deleted by $c_{j}$ equals the element inserted by $e_{j-1}$.

As for partitions, the number of parts of a composition $\alpha$ will be written $\ell(\alpha)$ and called the length of $\alpha$.

Corollary 4.7. If $\sigma \in \mathfrak{S}_{n}(1243,1324,3124,4321)$ then $\ell(\alpha(\sigma)) \leqslant 5$.

Proof. Suppose, to the contrary, that $\ell(\alpha(\sigma)) \geqslant 6$. Removing all but the first 6 increasing runs of $\sigma$ and standardizing we can assume $\ell(\alpha(\sigma))=6$. Now applying the bijections $c_{j}$ from the previous proposition for various values of $j$, we obtain a $\tau \in \mathfrak{S}_{m}(1243,1324,3124,4321)$ that has no increasing run of length greater than 2. It follows that $m \leqslant 12$. But we have verified by computer that no such $\tau$ exists.

We now have all the tools in place to compute $Q_{n}(\Pi)$ where $\Pi$ is given by $(6)$ and just need one more definition. For compositions we will use the partial order $\alpha \leqslant \beta$ if $\ell(\alpha)=\ell(\beta)$ and $\alpha_{i} \leqslant \beta_{i}$ for all $i$. Note that we consider compositions of different lengths to be incomparable.

Theorem 4.8. Let $\Pi=\{1243,1324,3124,4321\}$. Then

$$
Q_{n}(\Pi)=\sum_{\lambda} f^{\bar{\lambda}} s_{\lambda}
$$

where the sum is over all $\lambda \in \mathcal{H}_{n, 3}$ with $\ell(\lambda) \leqslant 3$, and $\bar{\lambda}$ is $\lambda$ with $\lambda_{1}$ replaced by $\min \left\{\lambda_{1}, 2\right\}$. In particular, for $n \geqslant 6$ we have

$$
Q_{n}(\Pi)=s_{n}+2 s_{n-1,1}+2 s_{n-2,2}+3 s_{n-2,1,1}+5 s_{n-3,2,1}+5 s_{n-4,2,2} .
$$

Proof. The "in particular" follows from equation (7) and the fact that when $n \geqslant 6$ we always have $\lambda_{1}$ replaced by 2 in $\bar{\lambda}$.

We will prove (7) by induction on $n$, where we have verified it by computer for $n \leqslant 10$. Consider any nonempty set $A(\alpha, n)$ for $n \geqslant 11$. By Corollary 4.7 , the fact that this set is nonempty forces there to be a part $\alpha_{i} \geqslant 3$. Applying the contraction bijections from Proposition 4.6, we see that there is a $\beta \models 10$ such that $\# A(\beta, 10)=$ $\# A(\alpha, n)$. 
Now consider the sum on the right-hand side of (7), which, since $n \geqslant 11$, reduces to (8). Using Theorem 1.1, we see that we want to show that $Q_{n}(\Pi)$ equals

$$
\begin{aligned}
F_{n}+2 & {\left[\sum_{\alpha \geqslant(1,1)} F_{\alpha}+\sum_{\alpha \geqslant(2,2)} F_{\alpha}+\sum_{\alpha \geqslant(1,2,1)} F_{\alpha}\right]+3 \sum_{\alpha \geqslant(1,1,1)} F_{\alpha} } \\
+5 & {\left[\sum_{\alpha \geqslant(2,2,1)} F_{\alpha}+\sum_{\alpha \geqslant(2,2)} F_{1 \alpha}+\sum_{\alpha \geqslant(2,1,2)} F_{\alpha}+\sum_{\alpha \geqslant(1,2,1,1)} F_{\alpha}+\sum_{\alpha \geqslant(1,1,2,1)} F_{\alpha}\right.} \\
& \left.+\sum_{\alpha \geqslant(2,2,2)} F_{\alpha}+\sum_{\alpha \geqslant(1,2,1,2)} F_{\alpha}+\sum_{\alpha \geqslant(2,2,1)} F_{1 \alpha}+\sum_{\alpha \geqslant(2,1,2,1)} F_{\alpha} F_{\alpha \geqslant(2,2,2,1)} F_{\alpha}+\sum_{\alpha \geqslant(1,2,1,2,1)} F_{\alpha}\right]
\end{aligned}
$$

where $1 \alpha$ is the composition obtained by concatenating (1) and $\alpha$, and all subscripts of the $F$ 's are compositions of $n$. By way of illustration, to obtain $s_{n-1,1}$ we consider the $n-1$ SYT of shape $(n-1,1)$ and find that their descent sets have corresponding compositions $(1, n-1),(2, n-2), \ldots,(n-1,1)$. But these are exactly the $\alpha \models n$ with $\alpha \geqslant(1,1)$. So the associated quasisymmetric functions are those in the first sum of the previous displayed equation. The other summations are explained similarly.

To finish the induction, it suffices to show that the sums of fundamentals to which $\alpha$ contributes are the same as the ones to which $\beta$ contributes where $\alpha$ and $\beta$ are as in the previous paragraph. But all of the lower bounds in the sums are compositions with only ones and twos. And applying $c_{j}$ reduces a part of size at least three to a part of size at least two and leaves all other parts the same. Therefore this map does respect the expansion into fundamentals above and we are done.

Note that (8) shows that the Schur expansion for this set of patterns stabilizes as $n \rightarrow \infty$. We will have more to say about stability in the next and last sections.

\section{KnUth Classes}

The final entries from Table 1 that need to be explained are $\{132,312\}$ and $\{213,231\}$. The reader will recognize these as Knuth classes $K(P)$ for $P$ equal to

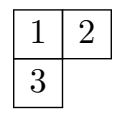

and

$$
\begin{array}{|l|l|}
\hline 1 & 3 \\
\hline 2 & \multicolumn{1}{|l}{,} \\
\cline { 1 - 1 }
\end{array}
$$

respectively. The main result of this section will be a characterization of the $P$ for which $\mathfrak{S}_{n}(K(P))$ is a union of Knuth classes for all $n$. For such $P$ it is clear that $Q_{n}(K(P))$ will be Schur nonnegative. We will first deal with the special case when $P$ is one of the two tableaux above.

We will concentrate on $\{132,312\}$ since the other $\Pi$ is just its reversal. It follows easily from the inductive description of the $\sigma \in \mathfrak{S}_{n}(132,312)$ in [15] that they are 
exactly the permutations such that for all $j \geqslant 2$ we have

$$
\sigma_{j}=\left\{\begin{array}{l}
\left(\min _{i<j}\left\{\sigma_{i}\right\}\right)-1, \quad \text { or } \\
\left(\max _{i<j}\left\{\sigma_{i}\right\}\right)+1 .
\end{array}\right.
$$

Equivalently, for all $j \geqslant 1$ the set $\left\{\sigma_{1}, \sigma_{2}, \ldots, \sigma_{j}\right\}$ is an interval of integers.

Proposition 5.1 ([7, Lemma 4.5]). For $n \geqslant 1$ we have

$$
Q_{n}(132,312)=Q_{n}(213,231)=\sum_{\lambda \in \mathcal{H}_{n}} s_{\lambda} .
$$

Proof. By reversal, it suffices to prove that $Q_{n}(132,312)$ is equal to the sum. As in the proof of Proposition 4.1, it suffices to show that Des restricts to a bijection $\mathfrak{S}_{n}(132,312) \rightarrow 2^{[n-1]}$. But given $J \subseteq[n-1]$ there is a unique $\sigma$ avoiding $\{132,312\}$ with that descent set, which can be constructed as follows. Arrange the integers $1,2, \ldots, \# J$ in a decreasing sequence in the positions of $J+1$ (the set obtained by adding one to every element of $J$ ) of $\sigma$. Then arrange the integers $\# J+1, \# J+2, \ldots, n$ as an increasing sequence in the remaining positions. It is easy to check that the permutation thus constructed has $\operatorname{Des} \sigma=J$.

We now consider general Knuth classes $K(P)$. We first need to recall some general facts about these sets. Suppose we have positive integers $a<b<c$ and a permutation $\sigma$ having a factor (subsequence of consecutive elements) $a c b$ or $c a b$. Then we can perform a Knuth move on $\sigma$ by exchanging one factor for the other. Alternatively, a Knuth move can exchange factors of the form $b a c$ and $b c a$. Two permutations are Knuth equivalent if one can be transformed into the other by a sequence of Knuth moves. For a proof of the next result, see [13].

TheOREM 5.2. If $P(\sigma)=P$ then $K(P)$ is the set of permutations Knuth equivalent to $\sigma$.

Call $\Pi$ pattern-Knuth closed if $\mathfrak{S}_{n}(\Pi)$ is a union of Knuth classes for all $n$. Equivalently, the complement $\overline{\mathfrak{S}}_{n}(\Pi)$ is a union of Knuth classes for all $n$. The following lemma is easy to prove directly from the definitions so its demonstration is omitted.

LEMmA 5.3. If $\Pi$ is pattern-Knuth closed then so are $\Pi^{c}, \Pi^{r}$, and $\Pi^{r c}$.

There is a sense in which this property is stable.

LEMMA 5.4. The set $\Pi$ is pattern-Knuth closed if and only if $\mathfrak{S}_{n}(\Pi)$ is a union of Knuth classes for $n \leqslant M+1$ where $M$ is the maximum length of a permutation in $\Pi$.

Proof. The forward direction is obvious so we will prove the converse. It will be more convenient to show that $\overline{\mathfrak{S}}_{n}(\Pi)$ is a union of Knuth classes for $n>M+1$. Suppose $\sigma \in \overline{\mathfrak{S}}_{n}(\Pi)$ contains a copy $\kappa$ of some $\pi \in \Pi$. By Theorem 5.2 , it suffices to show that the result $\sigma^{\prime}$ of performing a Knuth move on $\sigma$ will still be in $\overline{\mathfrak{S}}_{n}(\Pi)$. We will do this for a factor $a c b$ where $a<b<c$ as the other possible Knuth moves can be handled similarly. There are three cases.

If $\kappa$ does not contain both $a, c$ then $\kappa$ is still a subsequence of $\sigma^{\prime}$ and we are done. If $\kappa$ contains all three elements, then $\sigma^{\prime}$ contains $\kappa^{\prime}$, which is formed by replacing acb by $c a b$ in $\kappa$. Since our hypothesis implies that $\Pi$ is a union of Knuth classes, $\kappa^{\prime}$ is a copy of an element of $\Pi$ and we are again finished. The final case is when $a, c$ are in $\kappa$ but $b$ is not. Let $\pi^{\prime}$ be the standardization of the subsequence of $\sigma$ containing $\kappa$ and $b$. Then $\pi^{\prime} \in \overline{\mathfrak{S}}_{n}(\Pi)$ for some $n \leqslant M+1$. It follows from the hypothesis in this direction that the permutation $\pi^{\prime \prime}$ obtained by switching the elements corresponding to $a, c$ in $\pi^{\prime}$ still contains an element of $\Pi$. Since $\sigma^{\prime}$ contains a copy of $\pi^{\prime \prime}$ this finishes the proof. 
We now show that being pattern-Knuth closed is preserved by shuffling.

Lemma 5.5 ([7, Theorem 2.5]). If $A \subseteq \mathfrak{S}_{m}$ and $B \subseteq \mathfrak{S}_{n}$ are unions of Knuth classes then so is $A \amalg B$.

Proof. We will show that if $\alpha \in A, \beta \in B$ and $\sigma \in \alpha ш \beta$ contains the factor acb where $a<b<c$, then replacing this factor by $c a b$ results in $\sigma^{\prime} \in A \amalg B$. The proof for the other Knuth moves is similar. There are three cases.

If $a \leqslant m<c$ then $\alpha$ and $\beta+m$ are still subwords of $\sigma^{\prime}$ and so we are done. If $c \leqslant m$ then $a c b$ is a factor of $\alpha$. But $A$ is a union of Knuth classes so replacing this factor by $c a b$ gives $\alpha^{\prime} \in A$. Thus $\sigma^{\prime} \in \alpha^{\prime} ш \beta \subseteq A ш B$. The only other possibility is $a>m$ in which case a similar argument to the one just given with $A$ replaced by $B$ completes the proof.

Combining this lemma and equation (2) we immediately see that Conjecture 3.4 is true in the case when $\Pi$ and $\Pi^{\prime}$ are pattern-Knuth closed. In fact, we only need to assume that one of $\Pi$ and $\Pi^{\prime}$ is pattern-Knuth closed.

Proposition 5.6. If $\Pi$ and $\Pi^{\prime}$ are pattern-Knuth closed then so is $\Pi \varpi \Pi^{\prime}$. If $Q_{n}(\Pi)$ is Schur nonnegative for all $n$ and $\Pi^{\prime}$ is pattern-Knuth closed, then $Q_{n}\left(\Pi \amalg \Pi^{\prime}\right)$ is Schur nonnegative for all $n$.

Proof. The first sentence follows from Lemma 5.5 and equation (2). Under the hypotheses of the second sentence, Theorem 3.1 implies that $Q_{n}\left(\Pi \amalg \Pi^{\prime}\right)$ will be Schur nonnegative for all $n$ if the same is true of $s_{1} Q_{n-1}\left(\Pi^{\prime}\right)-Q_{n}\left(\Pi^{\prime}\right)$. Recalling the homomorphism $\Phi: \mathrm{MR} \rightarrow$ QSym sending $w \mapsto F_{w}$ from the proof of Theorem 3.1, we have

$$
s_{1} Q_{n-1}\left(\Pi^{\prime}\right)-Q_{n}\left(\Pi^{\prime}\right)=\Phi\left(\sum_{w \in A} w\right)
$$

where $A=\left(\mathfrak{S}_{n-1}\left(\Pi^{\prime}\right) ш \iota_{1}\right) \backslash \mathfrak{S}_{n}\left(\Pi^{\prime}\right)$. But $A$ is a union of Knuth classes by Lemma 5.5 and the assumption that $\Pi^{\prime}$ is pattern-Knuth closed.

We need one more result before we prove the main theorem of this section. For $J \subseteq[n-1]$ we let

$$
D_{J}=\left\{\pi \in \mathfrak{S}_{n} \mid \operatorname{Des} \pi=J\right\} .
$$

Suppose that $\operatorname{RS}(\pi)=(P, Q)$. Then, by Theorem $2.2(1)$, we have $\pi \in D_{J}$ if and only if $\operatorname{Des} Q=J$. As with other operations, we apply the inverse operator to a set of permutations by applying it to each individual permutation. It now follows from what we have just said and Theorem 2.2 (4) that $\pi \in D_{J}^{-1}$ if and only if $\operatorname{Des} P=J$. Then $D_{J}^{-1}$ is a union of Knuth classes. Also, it follows easily from the definitions that $\operatorname{Des}\left(\pi^{-1}\right)$ is the set of all $i$ such that $i+1$ appears to the left of $i$ in $\pi$. These two observations are important for the proof of the following result.

LEMma 5.7. For any $J$, the set $D_{J}^{-1}$ is pattern-Knuth closed.

Proof. The proof is very similar to that of Lemma 5.4. We only need to take some care with the last case for which we use the same notation as in that demonstration. Suppose first that the elements of $\pi$ corresponding to $a, c$ in $\kappa$ are not consecutive in value. From the discussion before the lemma, switching them will result in $\kappa^{\prime}$ whose standardization has the same inverse descent set as $\kappa$, namely $J$. Thus $\sigma^{\prime} \in \overline{\mathfrak{S}}_{n}\left(D_{J}^{-1}\right)$. Now suppose that $a$ and $c$ standardize respectively to $i$ and $i+1$ in $\pi$. It follows that the other elements of $\kappa$ are all less than $a$ or greater than $c$. Now let $\kappa^{\prime}$ be $\kappa$ with $c$ replaced by $b$. Since $a<b<c$ we have that $\kappa^{\prime}$ also standardizes to $\pi$, and $\kappa^{\prime}$ is a subsequence of $\sigma^{\prime}$, finishing this subcase. 
To state our principal result, we need one last set of definitions. The row superstandard Young tableau of shape $\lambda$ is the SYT obtained by filling the first row with $1,2 \ldots, \lambda_{1}$; the next row with $\lambda_{1}+1, \lambda_{1}+2, \ldots, \lambda_{1}+\lambda_{2}$; and so on. Note that the tableau in (9) is row superstandard. A column superstandard Young tableau is defined similarly except that one fills the columns from left to right; see (10). A tableau of either type is just called superstandard

TheOREM 5.8. The class $K(P)$ is pattern-Knuth closed if and only if $P$ is a superstandard tableau of hook shape.

We will prove this in a sequence of propositions which deal with the various cases involved. Note that because of Theorem $2.2(3)$ and Lemma 5.3 we have $K(P)$ is pattern-Knuth closed if and only if $K\left(P^{t}\right)$ is. Therefore we only need to prove closure, or lack thereof, for one of $P$ or $P^{t}$. We also assume throughout that the shape of $P$ is $\lambda \vdash n$.

Proposition 5.9. If $P$ is superstandard of hook shape then $K(P)$ is pattern-Knuth closed.

Proof. Suppose $\lambda=\left(n-k, 1^{k}\right)$. By the remarks just before this proposition it suffices to prove this when $P$ is column superstandard. It is easy to see that in this case $K(P)=D_{J}^{-1}$ where $J=[k]$. The result then follows from Lemma 5.7.

It will be convenient in our proofs to work with permutations and SYT having elements that are rational numbers, not just integers. To this end, given an integer $a$ we let $a^{+}=a+1 / 2$ and $a^{-}=a-1 / 2$. It will be important when comparing such permutations and tableaux that we always standardize them to have entries $[n]$ for some $n$. We will also need the column reading word of an SYT $P$, which is the permutation $\rho(P)$ obtained by recording the elements in each column of $P$ read bottom to top and then concatenating the sequences for the columns left to right. It is easy to see that the insertion tableau of $\rho(P)$ is $P$.

Proposition 5.10. If $P$ is of hook shape but not superstandard then $K(P)$ is not pattern-Knuth closed.

Proof. Our strategy, as suggested by Lemma 5.4, will be to find an element $\sigma \in$ $\overline{\mathfrak{S}}_{n+1}(K(P))$ to which a Knuth move can be applied creating a permutation $\sigma^{\prime}$ having no subsequence $\kappa$ with std $P(\kappa)=P$. By transposition if necessary, we can assume that $n$ is in the first column of $P$. Since $P$ is not superstandard, there is some $a>1$ in its first column such that $a+1$ is in the first row of $P$. Let $a$ be the largest such element. Let $b>a+1$ be the next element in $P$ 's first column so that we have the following situation

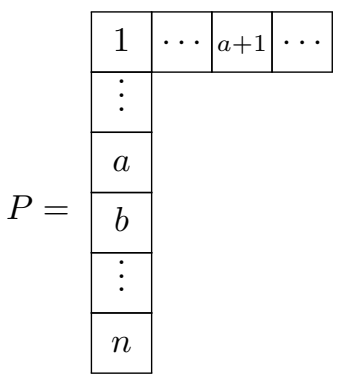

with $b+1$ being in the first column if $b<n$.

Let $\sigma$ be the permutation obtained by placing $a^{+}$just before $b \in \rho(P)$ so that

$$
\sigma=n, \ldots, a^{+}, b, a, \ldots
$$


which by construction is an element of $\overline{\mathfrak{S}}_{n+1}(K(P))$. Now exchange $a$ and $b$ to obtain

$$
\sigma^{\prime}=n, \ldots, a^{+}, a, b, \ldots
$$

Knuth equivalent to $\sigma$. Let $\kappa$ be any subsequence of $\sigma^{\prime}$ with $|\kappa|=n$ and suppose $k$ is the element removed to form $\kappa$. If $k=b$ then the first column of $P(\kappa)$ contains $a^{+}$, which standardizes to $a+1$ so that std $P(\kappa) \neq P$. If $k \neq b$ then when $b$ is inserted it will enter the tableau in box $(1,2)$ since either $a$ or $a^{+}$will be in box $(1,1)$ at that point. When $P(\kappa)$ is standardized, $b$ will either stay the same or be replaced by $b+1$. But both those elements need to be in the first column, so at some point $b$ will be bumped out of the first row. But then it will enter the second row in box $(2,2)$ because at least two of $1, a, a^{+}$are in $\kappa$ so that the element in box $(2,1)$ when $b$ is bumped will be $a$ or smaller. Then there will be a $(2,2)$ entry in $P(\kappa)$ forcing its shape not to be a hook, which completes our proof.

Note that by performing transpositions, it suffices to prove Theorem 5.8 for tableaux $P$ satisfying $P_{2,1}=2$.

Proposition 5.11. Let $P$ be a standard tableau of non-hook shape with $P_{2,1}=2$. If there exists an $i$ with $P_{i, 1}>P_{i-1,2}$ then $K(P)$ is not pattern-Knuth closed.

Proof. We will use the strategy and notation of the proof of the previous proposition. We can write $P$ in the following form

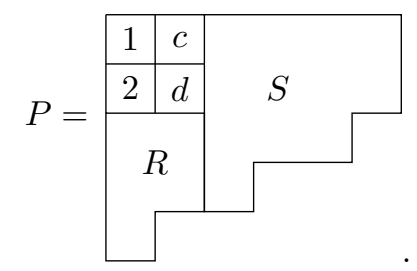

It is easy to see that the insertion tableau of $\pi=\rho(R), 2, d, 1, c, \rho(S)$ is $P$. We consider

$$
\sigma=\rho(R), 2, d, 2^{+}, 1, c, \rho(S),
$$

which is Knuth equivalent to

$$
\sigma^{\prime}=\rho(R), d, 2,2^{+}, 1, c, \rho(S) .
$$

Remove an element $k$ from $\sigma^{\prime}$ to form $\kappa$. There are two cases.

Suppose first that $k \notin S_{1} \cup\left\{2,2^{+}, c\right\}$ where $S_{1}$ is the first row of tableau $S$. It follows that $2,2^{+}, c, S_{1}$ is an increasing subsequence of $\kappa$ of length $\lambda_{1}+1$ where $\operatorname{sh} P=\lambda$. Thus, by Theorem $2.2(2), P(\kappa)$ has first row of length longer than $\lambda_{1}$ and so is not of the correct shape. If $k \in S_{1} \cup\left\{2,2^{+}, c\right\}$ then $k \neq 1, d$. Let $i$ be an index with $P_{i, 1}>P_{i-1,2}$ and note that $i \geqslant 3$ so that $P_{i-1,2} \geqslant d$. It follows that $P_{\ell(\lambda), 1}, \ldots, P_{i, 1}, P_{i-1,2}, \ldots, P_{3,2}, d, x, 1$ is a decreasing subsequence of $\kappa$ where $x$ is either 2 or $2^{+}$. But this subsequence has length $\ell(\lambda)+1$. If follows from Theorem $2.2(2)$ and (3) that $P(\kappa)$ will have first column of length longer than $\ell(\lambda)+1$ and so, again, will not have the right shape. This finishes the proof.

We can extend the definition of $P$ by letting $P_{i, j}=\infty$ for $i, j \geqslant 1$ and $(i, j)$ outside $\lambda=\operatorname{sh} P$. In this case, the proof of the previous proposition still goes through in the case where $P$ 's first two columns are of equal length since $P_{\ell(\lambda)+1,1}=\infty>P_{\ell(\lambda), 2}$. We can now finish the proof of Theorem 5.8 with the following proposition.

Proposition 5.12. Let $P$ be of non-hook shape. If $P_{i+1,1}<P_{i, 2}$ for all $1 \leqslant i \leqslant t$ where $t$ is the length of $P$ 's second column, then $K(P)$ is not pattern-Knuth closed. 
Proof. We can write

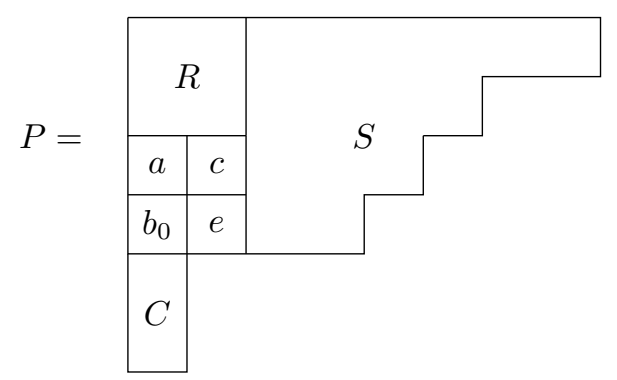

where $C$ is a single column. Note that by the discussion just before this proposition, $C$ must be nonempty. Let $\rho(C)=f_{p} \ldots f_{1} d_{l} \ldots d_{1} b_{k} \ldots b_{1}$ where

$$
a<b_{0}<b_{1}<\cdots<b_{k}<c<d_{1}<\cdots<d_{l}<e<f_{1}<\cdots<f_{p} .
$$

Note that either $k>0$ or $l>0$ since $C$ must contain an element less than $e$ to satisfy the hypothesis of this proposition. Our proof splits into two cases.

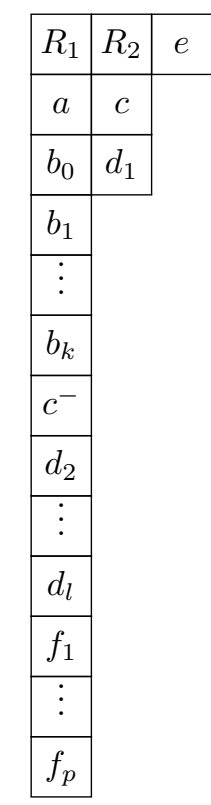

Figure 1. The insertion tableau for $\sigma^{\prime}$ in Case 1.

CASE $1 . l>0$. Define

$$
\pi=\rho(C), b_{0}, e, a, c, \rho(R), \rho(S) .
$$

It easy to check that $P(\pi)=P$. Also let

$$
\sigma=f_{p}, \ldots, f_{1}, d_{l}, \ldots, d_{2}, c^{-}, d_{1}, b_{k}, \ldots, b_{0}, e, a, c, \rho(R), \rho(S)
$$

which is obtained from $\pi$ by adding $c^{-}$. Apply a Knuth move to obtain

$$
\sigma^{\prime}=f_{p}, \ldots, f_{1}, d_{l}, \ldots, d_{2}, c^{-}, b_{k}, d_{1}, b_{k-1}, \ldots, b_{0}, e, a, c, \rho(R), \rho(S)
$$

Remove any $h$ from $\sigma^{\prime}$ to obtain $\kappa$. We will show $P(\kappa) \neq P$.

Consider the insertion tableau of $\sigma$ up to and including $\rho(R)$, which will have the form given in Figure 1 where $R_{1}$ and $R_{2}$ are the two columns of $R$. If $h$ comes from the first column of this tableau then its removal will cause all the entries to shift up 
by one, making the first column too short. The only way to compensate for this is if the insertion of $\rho(S)$ causes $e$ to bump into the first column in row $r$ just below $b_{0}$. But then

$$
P(\kappa)_{r, 1}=e>d_{1}=P(\kappa)_{r-1,2}
$$

which is a contradiction. On the other hand, if $h$ comes from the second column of Figure 1 then $e$ will have to end up in the second column to preserve column lengths. And if $h$ comes from a column of $S$ then the first two columns in the figure must remain undisturbed. In either case the first column will contain more entries in the interval $\left[b_{0}, c\right]$ than $P$ does, which finishes this case.

CASE $2 . l=0$ and $k>0$. Now we consider

$$
\pi=f_{p}, \ldots, f_{1}, b_{k}, e, b_{k-1}, \ldots, b_{0}, a, c, \rho(R), \rho(S) .
$$

It is easily checked that since $k>0$ we have $P(\pi)=P$. Add $c^{+}$to obtain

$$
\sigma=f_{p}, \ldots, f_{1}, c^{+}, b_{k}, e, b_{k-1}, \ldots, b_{0}, a, c, \rho(R), \rho(S)
$$

and perform a Knuth move to yield

$$
\sigma^{\prime}=f_{p}, \ldots, f_{1}, c^{+}, e, b_{k}, b_{k-1}, \ldots, b_{0}, a, c, \rho(R), \rho(S) .
$$

Define $h, \kappa$, and $R_{1}$ as before with the goal of showing $P(\kappa) \neq P$.

Note that there is a decreasing subsequence of $\sigma^{\prime}$ of length $\ell(\lambda)+1$ consisting of the $f^{\prime}$ 's, either $c^{+}$or $e$, the $b$ 's, $a$, and $\rho\left(R_{1}\right)$. Therefore $h \in\left\{a, b_{0}, \ldots, b_{k}, f_{1}, \ldots, f_{p}\right\} \cup R_{1}$. If $h$ is one of the $f$ 's then $P(\kappa)$ will have more elements in the interval $[a, e]$ in its first column than $P$. If $h$ is any of the other possibilities, then $P(\kappa)$ will have more elements greater than $c$ in its first column than $P$. Either way, we have our final contradiction.

We conclude this section with two questions. First of all, we know from Lemma 2.3 and Theorem 5.8 that when $P$ is superstandard of hook shape then $Q_{n}(K(P))$ is Schur nonnegative.

QUESTION 5.13. Find a combinatorial interpretation for the coefficients in the Schur expansion for $Q_{n}(K(P))$ when $P$ is superstandard of hook shape.

It is also natrual to ask about generalizing Theorem 5.8 to pairs of tableaux.

Question 5.14. Let $P, Q$ be standard Young tableaux. Find a necessary and sufficient condition for $K(P) \cup K(Q)$ to be pattern-Knuth closed.

We note that if $\Pi, \Pi^{\prime}$ are both pattern-Knuth closed then so is $\Pi \cup \Pi^{\prime}$ since in this case $\overline{\mathfrak{S}}_{n}\left(\Pi \cup \Pi^{\prime}\right)$ is the union of the Knuth classes of $\overline{\mathfrak{S}}_{n}(\Pi)$ and $\overline{\mathfrak{S}}_{n}\left(\Pi^{\prime}\right)$. This together with Theorem 5.8 shows that if $P, Q$ are superstandard hooks then $K(P) \cup K(Q)$ is pattern-Knuth closed. We also note that both of these questions have been answered by Bloom and Sagan [2]. In particular, they were able to deal with Question 5.14 by giving a more conceptual proof of Theorem 5.8 .

\section{Arc permutations}

Elizalde and Roichman introduced and studied arc permutations in [4, 5]. They are closely related to two of the permutation classes we have been considering and so we will be able to produce a bijection between the arc permutations and permutations avoiding a certain shuffle.

A permutation $\sigma \in \mathfrak{S}_{n}$ is in the set of arc permutations, $\mathcal{A}_{n}$, if each prefix $\sigma_{1} \sigma_{2} \ldots \sigma_{i}$ is an interval in the integers modulo $n$. Equivalently, $\mathcal{A}_{n}=\mathfrak{S}_{n}\left(\Pi_{\mathcal{A}}\right)$ where

$$
\Pi_{\mathcal{A}}=\{1324,1342,2413,2431,3124,3142,4213,4231\} .
$$


Recall that the permutations avoiding $\{132,312\}$ were those where each prefix was an interval of integers and a similar statement is true for suffixes when avoiding $\{213,231\}$. Define the pin shaped permutations to be

$$
\mathcal{P}_{n}=\mathfrak{S}_{n}(132,312) \cup \mathfrak{S}_{n}(213,231) \subseteq \mathcal{A}_{n}
$$

(Elizalde and Roichman call these permutations unimodal because their rotations are unimodal sequences, but we prefer to reserve unimodal for its original meaning.) We will also be using the complement $\mathcal{Z}_{n}=\mathcal{A}_{n}-\mathcal{P}_{n}$.

In order to define the symmetric functions to describe $Q_{n}\left(\Pi_{\mathcal{A}}\right)$ we will need a few more definitions. Consider the set of non-trivial hooks

$$
\overline{\mathcal{H}}_{n}=\mathcal{H}_{n}-\left\{(n),\left(1^{n}\right)\right\}
$$

as well as the diagrams one obtains from the elements of $\overline{\mathcal{H}}_{n}$ by adding the $(2,2)$ box

$$
\mathcal{T}_{n}=\left\{\lambda \cup(2,2) \mid \lambda \in \overline{\mathcal{H}}_{n-1}\right\} .
$$

Define the corresponding generating functions $\bar{H}_{n}=\sum_{\lambda \in \overline{\mathcal{H}}_{n}} s_{\lambda}$ and $T_{n}=\sum_{\lambda \in \mathcal{T}_{n}} s_{\lambda}$. The following result is a consequence of $[4$, Theorem 7.7].

TheOREM 6.1. For all $n \geqslant 0$ we have

$$
Q_{n}\left(\Pi_{\mathcal{A}}\right)=T_{n}+2 \bar{H}_{n}+s_{n}+s_{1^{n}} .
$$

There is a shuffle class with the same expansion. Let

$$
\Pi_{\mathcal{S}}=\{1\} \amalg\{132,312\}=\{1243,1423,2143,4123,2413,4213,2431,4231\} .
$$

Proposition 6.2. For all $n \geqslant 0$ we have

$$
Q_{n}\left(\Pi_{\mathcal{S}}\right)=Q_{n}\left(\Pi_{\mathcal{A}}\right)
$$

Proof. Applying Theorem 3.1 with $\Pi=\{1\}$ and $\Pi^{\prime}=\{132,312\}$, then Proposition 5.1, and finally the Pieri formula gives

$$
Q_{n}\left(\Pi_{S}\right)=Q_{n}\left(\Pi^{\prime}\right)+s_{1} Q_{n-1}\left(\Pi^{\prime}\right)-Q_{n}\left(\Pi^{\prime}\right)=T_{n}+2 \bar{H}_{n}+s_{n}+s_{1^{n}} .
$$

Comparing this with the previous result completes the proof.

Note that by reversal, complement, and Proposition 4.1, the previous proposition is true if $\Pi_{\mathcal{S}}$ is replaced by either $\{1\} ш \Pi$ or $\Pi \varpi\{1\}$ for any

$$
\Pi \in\{\{132,312\},\{132,213\},\{213,231\},\{231,312\}\} .
$$

However, there is no dihedral symmetry relating any of these shuffles to $\Pi_{\mathcal{S}}$.

Elizalde and Roichman [4, Section 7.4] gave a bijective proof of Theorem 6.1. In particular, they proved the following result.

THEOREM 6.3. For all $n \geqslant 0$, there is an explicit bijection

$$
\phi: \mathcal{Z}_{n} \rightarrow \bigcup_{\lambda \in \mathcal{T}_{n}} \operatorname{SYT}(\lambda)
$$

As a consequence, we have the following.

Corollary 6.4. For all $n \geqslant 0$, there is an explicit bijection

$$
\psi: \mathfrak{S}_{n}\left(\Pi_{\mathcal{A}}\right) \rightarrow \mathfrak{S}_{n}\left(\Pi_{\mathcal{S}}\right) .
$$

Proof. Note that by their descriptions in terms of prefixes and suffixes, we have

$$
\mathfrak{S}_{n}(132,312) \cap \mathfrak{S}_{n}(213,231)=\left\{\iota_{n}, \delta_{n}\right\} .
$$

For $\sigma \in \mathfrak{S}_{n}\left(\Pi_{\mathcal{A}}\right)$ we define $\psi(\sigma)$ as follows, using $Q(\pi)$ as the Robinson-Schensted recording tableau.

(1) If $\sigma \in \mathfrak{S}_{n}(132,312)$ then let $\psi(\sigma)=\sigma$. 
(2) If $\sigma \in \mathcal{Z}_{n}$ then let $\psi(\sigma)=\tau$ where $\tau \in \mathfrak{S}_{n}\left(\Pi_{\mathcal{S}}\right)$ is the unique permutation with $Q(\tau)=\phi(\sigma)$.

(3) If $\sigma \in \mathfrak{S}_{n}(213,231)-\left\{\iota_{n}, \delta_{n}\right\}$ then let $\psi(\sigma)=\tau$ where $\tau \in \mathfrak{S}_{n}\left(\Pi_{\mathcal{S}}\right)-$ $\mathfrak{S}_{n}(132,312)$ is the unique permutation with $Q(\tau)=Q(\sigma)$.

Note that $(2)$ is well defined by Lemma 5.5 and the fact that $Q_{n}\left(\mathcal{Z}_{n}\right)$ is Schur multiplicity free. In a similar manner we see that (3) is well defined. The check that this is a bijection is now easily done.

The construction of the map $\psi$ is hardly as illuminating as one would hope.

QUESTION 6.5. Is there a direct description of a bijection between $\mathfrak{S}_{n}\left(\Pi_{\mathcal{A}}\right)$ and $\mathfrak{S}_{n}\left(\Pi_{\mathcal{S}}\right)$ on the level of permutations?

\section{Comments And open questions}

We end with some comments and some questions that we hope the reader will be interested in answering.

SyMmetry VS. NONNEGATIVITY. It is possible to construct $\Pi$ such that $Q_{n}(\Pi)$ is symmetric, but not Schur nonnegative. In particular, consider the following set of permutations where we have enclosed certain elements in parentheses for readability

$$
\begin{aligned}
X_{n}=\left\{\iota_{n}\right\} \cup & \{2314 \ldots n, 12 \ldots(n-3) n(n-2)(n-1)\} \\
& \cup\{2134 \ldots n, 1324 \ldots n, \ldots, 12 \ldots(n-2) n(n-1)\} \\
& \cup\{32145 \ldots n, 14325 \ldots n, \ldots, 12 \ldots(n-3) n(n-1)(n-2)\} .
\end{aligned}
$$

Let $\Pi=\mathfrak{S}_{4}-X_{4}$. Then one can verify that $\mathfrak{S}_{n}(\Pi)=X_{n}$ and so

$$
Q_{n}(\Pi)=s_{n}+2 s_{n-1,1}+s_{n-2,1,1}-s_{n-2,2} .
$$

STABILITY. The following is a natural question given the results we have proved such as Lemma 5.4.

QUESTION 7.1. Suppose $\Pi$ is nonempty and $M$ is the maximum length of a permutation in $\Pi$. Is there an $N$, a function of $M$, such that $Q_{n}(\Pi)$ symmetric for $n<N$ implies that it continues to be symmetric for $n \geqslant N$ ? What about the same question with "symmetric" replaced by "Schur nonnegative"?

It is worth pointing out that the converse of these questions is false. In particular, let $\lambda=(3,1,1)$ and

$$
P=\begin{array}{|l|l|l|}
\hline 1 & 2 & 4 \\
\hline 3 & \multicolumn{2}{|l}{} \\
\cline { 1 - 1 } 5 & \multicolumn{2}{|}{} \\
\hline
\end{array}
$$

Then Bloom and Sagan [2] have shown that if $\Pi=K(\lambda)-K(P)$ then $Q_{n}(\Pi)$ is Schur nonnegative for $n \geqslant 7$ but not even symmetric for $n=6$.

KNuth CLASSES. It would be interesting to determine when the union of Knuth classes is pattern-Knuth closed, generalizing Theorem 5.8. Of course, if $K$ and $L$ are pattern-Knuth closed then so is $K \cup L$. In the case that one gets a pattern-Knuth closed class $\Pi$, one would also like to characterize the coefficients in the Schur expansion of $Q_{n}(\Pi)$. In [2] this has been done in the case where $\Pi=K(P)$ for a superstandard hook tableau $P$. 
Representation theORY. Adin and Roichman [1] have developed a way to connect certain subsets of permutations with representations of $\mathfrak{S}_{n}$. Given $\alpha=\left(\alpha_{1}, \ldots, \alpha_{k}\right) \models$ $n$ and $\pi \in \mathfrak{S}_{n}$ the $\alpha$-decomposition of $\pi$ is the factorization $\pi=\pi^{(1)} \ldots \pi^{(k)}$ where $\# \pi^{(i)}=\alpha_{i}$ for all $i$. The $\alpha$-descent set and $\alpha$-descent number of $\pi$ are

$$
\operatorname{Des}_{\alpha} \pi=\bigcup_{i} \operatorname{Des} \pi^{(i)} \text { and } \operatorname{des}_{\alpha} \pi=\# \operatorname{Des}_{\alpha} \pi,
$$

respectively. An integer sequence $a_{1} \ldots a_{p}$ is comodal (complement unimodal) if, for some $m$, we have $a_{1}>\cdots>a_{m}<\cdots<a_{p}$. (Note that Adin and Roichman call such a sequence "unimodal" but that is at variance with standard practice.) Say that $\pi \in \mathfrak{S}_{n}$ is $\alpha$-comodal if each $\pi^{(i)}$ in its $\alpha$-decomposition is comodal. If $\Pi$ is a set of permutations then let $\Pi_{\alpha}$ denote the set of $\alpha$-comodal permutations in $\Pi$. Finally, call $\Pi \subseteq \mathfrak{S}_{n}$ fine if there is an $\mathfrak{S}_{n}$-character $\chi$ such that for all $\alpha \models n$

$$
\chi(\alpha)=\sum_{\pi \in \Pi_{\alpha}}(-1)^{\operatorname{des}_{\alpha} \pi}
$$

where $\chi(\alpha)$ is the value of $\chi$ on the conjugacy class indexed by $\alpha$.

Adin and Roichman give a number of conditions equivalent to a set of permutations $\Pi \subseteq \mathfrak{S}_{n}$ being fine, one of which is that $Q_{n}(\Pi)$ is symmetric and Schur nonnegative. This approach has been continued in the work of Elizalde and Roichman [6].

OTHER BASES. A couple of the generating functions we have calculated are Schur $P$-functions. For example, if $\Pi=\{132,312\}$ then $Q_{n}(\Pi)=P_{n}$ by Proposition 5.1, and $Q\left(\Pi_{\mathcal{A}}\right)=P_{n-1,1}$ by Theorem 6.1 .

Question 7.2. For which $\Pi$ is $Q_{n}(\Pi)$ Schur P-nonnegative for all $n$ ?

One could also change the set of quasisymmetric functions being used in $\mathrm{QSym}_{n}$. Let $\Theta_{\mathrm{Pk}}$ be the peak fundamental quasisymmetric function associated with a subset $\mathrm{Pk} \subseteq[2, n-1]$. Stembridge [17] developed a theory of enriched $P$-partitions ( $P$ here is a partially ordered set), which allowed him to prove an analogue of Theorem 1.1 where $s_{\lambda}$ is replaced by a Schur $P$-function and the $F_{\text {Des } P}$ are replaced by $\Theta_{\operatorname{Pk}(\sigma)}$ for certain permutations $\sigma$, where $\operatorname{Pk}(\sigma)$ is the peak set of $\sigma$. Schur $P$-functions are generating functions for certain shifted tableaux just as the usual Schur functions are for semistandard left-justified Young tableaux. Then it is natural to define for a set of permutations $\Pi$

$$
R_{n}(\Pi)=\sum_{\sigma \in \mathfrak{S}_{n}(\Pi)} \Theta_{\operatorname{Pk}(\sigma)}
$$

and ask the question

QUESTION 7.3. For which $\Pi$ is $R_{n}(\Pi)$ symmetric? In that case when is it Schur $P$ nonnegative?

Acknowledgements. We would like to thank the following people for helpful discussions: Alex Burstein, Vic Reiner, Yuval Roichman, John Stembridge, and Damir Yeliussizov. We especially thank Alex Woo for the idea which inspired this paper. Joel Lewis suggested several ideas that were critical in arriving at our results in Section 5 .

\section{REFERENCES}

[1] Ron M. Adin and Yuval Roichman, Matrices, characters and descents, Linear Algebra Appl. 469 (2015), 381-418.

[2] Jonathan Bloom and Bruce E. Sagan, Revisiting patterns avoidance and quasisymmetric functions, preprint, https://arxiv.org/abs/1812.10738, 2018.

[3] Miklós Bóna, Combinatorics of permutations, Discrete Mathematics and its Applications (Boca Raton), Chapman \& Hall/CRC, Boca Raton, FL, 2004. 
[4] Sergi Elizalde and Yuval Roichman, Arc permutations, J. Algebr. Comb. 39 (2014), no. 2, 301334.

[5] Signed arc permutations, J. Comb. 6 (2015), no. 1-2, 205-234.

[6] _ On rotated Schur-positive sets, J. Comb. Theory, Ser. A 152 (2017), 121-137.

[7] Schur-positive sets of permutations via products and grid classes, J. Algebr. Comb. 45 (2017), no. 2, 363-405.

[8] Paul Erdős and George Szekeres, A combinatorial problem in geometry, Compos. Math. 2 (1935), 463-470.

[9] Ira M. Gessel, Multipartite P-partitions and inner products of skew Schur functions, in Combinatorics and algebra (Boulder, Colo., 1983), Contemp. Math., vol. 34, Amer. Math. Soc., Providence, RI, 1984, pp. 289-317.

[10] Ian G. Macdonald, Symmetric functions and Hall polynomials, second ed., Oxford Mathematica Monographs, The Clarendon Press Oxford University Press, New York, 1995, With contributions by A. Zelevinsky, Oxford Science Publications.

[11] Claudia Malvenuto and Christophe Reutenauer, Duality between quasi-symmetric functions and the Solomon descent algebra, J. Algebra 177 (1995), no. 3, 967-982.

[12] Gilbert de B. Robinson, On the representations of the symmetric group, Am. J. Math. 60 (1938), no. 3, 745-760.

[13] Bruce E. Sagan, The symmetric group: Representations, combinatorial algorithms, and symmetric functions, second ed., Graduate Texts in Mathematics, vol. 203, Springer-Verlag, New York, 2001.

[14] Craige E. Schensted, Longest increasing and decreasing subsequences, Can. J. Math. 13 (1961), 179-191.

[15] Rodica Simion and Frank W. Schmidt, Restricted permutations, Eur. J. Comb. 6 (1985), no. 4, 383-406.

[16] Richard P. Stanley, Enumerative combinatorics. Vol. 2, Cambridge Studies in Advanced Mathematics, vol. 62, Cambridge University Press, Cambridge, 1999, With a foreword by Gian-Carlo Rota and appendix 1 by Sergey Fomin.

[17] John R. Stembridge, Enriched P-partitions, Trans. Am. Math. Soc. 349 (1997), no. 2, $763-788$.

Zachary Hamaker, University of Florida, Department of Mathematics, Gainesville, FL 326111941, USA

E-mail : zhamaker@ufl.edu

Brendan Pawlowski, University of Southern California, Department of Mathematics, Los Angeles, CA 90089-2532, USA

E-mail : bpawlows@usc.edu

Bruce E. Sagan, Michigan State University, Department of Mathematics, East Lansing, MI 488241027, USA

E-mail : sagan@math.msu.edu 